\title{
BMJ Open Deprescribing tool for use in older Australians with life-limiting illnesses and limited life expectancy: a modified- Delphi study protocol
}

\author{
Shakti Shrestha (D) , ${ }^{1}$ Arjun Poudel (D) , ${ }^{2}$ Kathryn J Steadman (D) , \\ Lisa M Nissen (i) ${ }^{2}$
}

To cite: Shrestha S,

Poudel A, Steadman KJ, et al. Deprescribing tool for use in older Australians with lifelimiting illnesses and limited life expectancy: a modified-Delphi study protocol. BMJ Open 2021;11:e043766. doi:10.1136/ bmjopen-2020-043766

- Prepublication history and supplemental material for this paper is available online. To view these files, please visit the journal online (http://dx.doi. org/10.1136/bmjopen-2020043766).

Received 13 August 2020 Revised 15 February 2021 Accepted 05 March 2021
Check for updates

(C) Author(s) (or their employer(s)) 2021. Re-use permitted under CC BY-NC. No commercial re-use. See rights and permissions. Published by BMJ.

${ }^{1}$ School of Pharmacy, The University of Queensland, Brisbane, Queensland, Australia ${ }^{2}$ School of Clinical Sciences, Queensland University of Technology, Brisbane, Queensland, Australia

Correspondence to Shakti Shrestha; shakti.shrestha@uq.edu.au

\section{ABSTRACT}

Background Making a meaningful decision on deprescribing of potentially inappropriate medications in older adults with lifelimiting illnesses (LLIS) and limited life expectancy (LLE) is often challenging. Therefore, we aimed to elicit opinion and gain consensus on a deprescribing tool for use in this population. Methods and analysis A modified-Delphi method will be used to obtain a consensus from a panel of experts in geriatric therapeutics on a deprescribing tool for use in people aged $\geq 65$ years with LLIs and LLE. Through an online survey, in the initial round, the panel will anonymously elicit their opinion on a series of items related to the conceptual model of the deprescribing tool, its practicality and deprescribing of medications, while on the controlled feedback in subsequent rounds till a consensus is reached or the panellists stop revising their answers. In each round, panel members will be using a 5-point Likert scale to rate their agreement with the statement. Consensus will be considered on $\geq 75 \%$ of agreement on the statements.

Ethics and dissemination All the participants will receive an invitation and participant information but they need to consent for the participation. Ethics approval has been granted from the University of Queensland Health and Behavioural Sciences, Low and Negligible Risk Ethics Sub-Committee (reference: 2020001069). The results of this project will be disseminated through conferences and a peer-reviewed clinical journal.

\section{INTRODUCTION}

Older people make up a considerable proportion in the Australian population. In 2017, over one in seven people were aged $\geq 65$ and over, and this population is anticipated to rise significantly as the remaining two-thirds of the postwar baby boom over the next decade. ${ }^{12}$ The leading causes of deaths in older Australians are coronary heart disease, dementia and Alzheimer disease, cerebrovascular disease, chronic obstructive pulmonary disease and cancer $^{23}$; the progression to advanced stage referred to as lifelimiting illnesses (LLIs).

Data from palliative care services in Australia state that in the year 2017-2018 about 1.1 million palliative care-related prescriptions were supplied to more than half a million patients
Strengths and limitations of this study

- The deprescribing tool for use in older adults with life-limiting illnesses and limited life expectancy will be validated by an interdisciplinary expert panel using a widely accepted consensus process.

- This study will collate information from existing knowledge and experience from experts in the geriatric field to make a meaningful decision on deprescribing in older population at end of life with life-limiting illnesses.

- Gaining consensus through a Delphi technique in itself will not result in a novel evidence and therefore, the tool needs to be tested in a clinical setting.

nationally, $35.7 \%$ were aged 65 and older. ${ }^{4}$ Though there is limited research in Australia in older adults with LLI and limited life expectancy (LLE), evidence in different settings have suggested that they continue to receive medications that are considered inappropriate. ${ }^{56}$ These inappropriate medications are continued even in the last few months of their life. ${ }^{7}$ This vulnerable population possess high risk of drugrelated adverse events because of the altered physiological system and the pharmacology of medicines. ${ }^{89}$ Moreover, with the progression of disease towards the end-of-life (EOL), medicine that was once clinically beneficial for prevention of disease can become less relevant. In all situations, it is important to achieve the EOL priorities of patients, one of which is maintaining a quality of life. ${ }^{1011}$ It is, therefore, essential to be considerate of the impact of all the medicines and whether it is necessary to withdraw medicines that are inappropriate for use in such patients at the EOL.

A potential approach to this could be deprescribing, a process of withdrawal (or dose reduction) of an inappropriate medication, supervised by a healthcare professional and hence a part of good clinical care. ${ }^{12}$ 
Evidence suggests that older adults and their caregivers in Australia are willing to have one of their or their care recipient's medication deprescribed. ${ }^{13}$ However, prescribers believe that the existing deprescribing guidelines are complex, often time-consuming, and they lack resources to make a meaningful decision in their daily practice. $^{1415}$

Moreover, the existing explicit criteria for medication optimisation developed for older people do not account for the additional complexities of patients with LLIs. This demands for an efficient deprescribing tool, specifically for use in older adults with LLIs and LLEs. Therefore, in this study, we aimed to elicit opinion and gain consensus on a deprescribing tool for use in older adults with LLIs and LLEs.

\section{METHODS AND ANALYSIS}

\section{Patient and public involvement statement}

This study does not require the involvement of patient and public.

\section{Study design and its justification}

A modified-Delphi technique will be used. This will apply an online survey to reach a consensus on the concept of a deprescribing tool developed by the research team. Delphi is a well-accepted technique to obtain consensus on study matter ${ }^{16}$ and has shown to have face, construct and predictive validity in observational studies. ${ }^{17}$ It has been used to develop and validate several tools for use in older adults such as explicit criteria to identify potentially inappropriate medications, ${ }^{18}{ }^{19}$ identification of priority medications for deprescribing, ${ }^{20}$ explicit criteria for deprescribing. ${ }^{21-24}$ The modified version involving a survey, as intended in the present study, has been successfully conducted earlier. ${ }^{20}{ }^{23-25}$ We plan to consider the online survey over a focused group discussion due to its practicality and cost-effectiveness as the panel will be located in diverse geographical locations. ${ }^{26}$ Additionally, the online survey will involve a 'controlled feedback' to provide the panel with a summary of comments and descriptive statistics of entire panel position ${ }^{16}$ with anonymity to each other. The anonymity will avoid individual dominance over the discussion while allowing each panel to determine their response relative to the overall group for providing a revision of their ranking in subsequent Delphi rounds. ${ }^{16}$

\section{Research team}

The research team comprise of the pharmacists, clinical pharmacologist and experts in older adults. The research team will prepare and circulate the content of the Delphi rounds. The team developed a conceptual model of a deprescribing tool for use in older adults at EOL but will not be participating in the survey, instead supervise and monitor the Delphi process.
Development of a conceptual model for the deprescribing tool The research team initially framed a conceptual model for the deprescribing tool based on earlier works on the deprescribing process ${ }^{27}$ used for medication review and our previous systematic review on outcomes of deprescribing intervention in older patients with LLIs and LLEs. ${ }^{28}$ The conceptual model consists of a three-step model for the deprescribing tool in line with the easyto-use concept for the tool in the clinical setting. It was pilot tested with two palliative care practitioners and their feedbacks were addressed to form the final conceptual model. The first step of the model is the evaluation of the risk and benefit of each medicine used by reflecting on the indication, contraindication, drug-drug interaction, adverse effects, cascade-prescribing, adherence to medicine, patient preference and potential future benefit provided the life expectancy. The second step use a traffic light concept to distinguish the medicine into one of the three types-preventive (red), dual-purpose (yellow) and symptom-control (green). The third step is on deprescribing each category of medicine through established process of deprescribing that may include a plan, regimen or algorithm. The survey will then undergo an external validation from a panel of experts via Delphi process.

\section{Selection and identification of the expert panel}

It is essential to have a non-homogeneous panel for the robustness of the technique. Therefore, the expert panel will consist of experts with a clinical or research background on geriatric medicine, geriatrics, family medicine or pharmacotherapy. This will include clinicians, pharmacists, nurses, pharmacologists and other identified relevant experts. To be eligible for the expert panel one or more of the criteria has to be met: practiced in a field relevant to medication optimisation for older adults for $\geq 5$ years postgraduation, or hold a postgraduate qualification or current credentials relevant to geriatrics (eg, a geriatrician, palliative care physician or a pharmacist credentialed with the certificate of geriatric pharmacy), or peers recognised expert in the field (eg, invitation to participate in a relevant symposium, focus group, conference or other event, or a member of a relevant organisation, or identified by our existing networks, or received a personalised invitation letter to participate in the study); or authored (first, second, last or corresponding) at least one peer-reviewed paper relevant to medication optimisation in older adults in the last 10 years. Those whose experience is not sufficient in the relevant field or those who would not be able to make a commitment of availability for the full process will be excluded.

\section{Recruitment and consent}

Participants will involve experts identified from practice and literature. Experts from practice will be identified through peers and relevant professional associations. Experts from the literature will be identified through the current publications in the field related to medicine use 
in older adults with LLIs and LLE, and published in the past 10 years. These authors will be contacted with an invitation to participate in the research. All the eligible experts identified from practice and literature will be sent an email invitation to participate in the project with a brief description of the study and a link directing to participant information. The participant information will provide a more detailed description of the study, the expected process, the commitment of participants over a period of time, possible benefits and risk associated with the survey, and the ethics-related information. On reading and understanding the information and having any questions answered to their satisfaction (if any), the invitee can consent to participate in the Delphi study by accepting the invitation.

\section{Survey development, data management and analysis}

The survey website will include an explanation of the project and Delphi round (online supplemental annex 1 on how to complete Delphi round 1, followed by a pictorial diagram and short explanation of the conceptual model for deprescribing in older adults with LLIs and LLEs. This will be followed by survey questions on the proposed deprescribing conceptual model for use in older adults with LLIs and LLEs (online supplemental annex 2 for indicative questions). In each round of the survey, information on the intention of the round will be stated and for the subsequent rounds, the feedback from the previous round will be provided. In each round, panel members will be asked to rate their agreement with the statements using a 5-point Likert scale (1=strongly disagree; $2=$ =agree; $3=$ neutral; $4=$ =agree; $5=$ =strongly agree) along with an additional choice to provide any comments or suggestions. The data will be automatically stored on the university server after the participants provide their response, which will be accessible only by members of the research team. Checkbox V.7 survey tool (Checkbox Survey, Massachusetts, USA) will be used for the online survey and the electronic data files will be stored indefinitely. The quantitative data (responses to the Likert scale) will be analysed using IBM-SPSS V.25.0 (IBM). For each statement, a consensus will be based on the median Likert response and IQR. A median value of 4-5 with a 25th percentile of $\geq 4$ will be acceptable for inclusion in the tool, that is, only those statements with at least $75 \%$ of respondents agreeing or strongly agreeing will be included. Any median values $\leq 3$ will be rejected but those median value of $4-5$ and a 25 th percentile of $<4$ will be rephrased based on the feedback from the panel to be included in the next round of the Delphi. The qualitative data (comments or suggestions on each statement) will be analysed using NVivo Qualitative Data Analysis Software V.10A (QSR International, Massachusetts, USA) to present back to the panellists as arguments for and against the set of statements in the next rounds and in the final results. This will allow the panellists to revise their rankings based on the 'controlled feedback' provided. The survey feedback will be continued until either consensus is reached or the panellists stop revising their answers. ${ }^{16}$

\section{Panel size and composition}

There is no universal agreement on the panel size for the Delphi technique. ${ }^{29}$ Though increasing panel size will increase the reliability of the consensus, ${ }^{29} 30$ having relevant and interested experts in the panel will be the priority. ${ }^{30}$ Therefore, we will include eligible experts with an intention to consider as many panellists as possible and from varying specialities.

\section{Anonymity}

All the members of the panel will be anonymous to each other but not to the researcher. ${ }^{31} 32$ At the start of the survey, they will have a brief explanation about the project, make it clear that they need not put any information that would identify them to the other members of the panel, and the data will be solely used for research.

\section{Enhancing response rate}

We will primarily aim to achieve a full response rate in each round but a minimum of $70 \%$ response rate in each round will be considered acceptable. Participants will be asked to complete the survey within 10 days after each round. A reminder will be sent on the seventh day. After 14 days the survey will be resent.

\section{Withdrawal}

The information provided to the participant in the participant information sheet and at the start of the survey will also inform them that they can choose to withdraw from the study at any stage during the completion of this survey and they can choose whether any of the data contributed up to that point can be used or should be destroyed.

\section{ETHICS AND DISSEMINATION}

The study will be conducted within the framework of ethical principles of the 'Declaration of Helsinki', Good Clinical Practice and within the laws and regulations of Australia. All the participants will receive an invitation and participant information, but they need to consent to participate. Anonymity will be maintained during the research. This study has been granted ethical approval by the University of Queensland Health and Behavioural Sciences, Low and Negligible Risk Ethics Sub-Committee (reference: 2020001069). The results of this project will be disseminated through conferences and a peerreviewed clinical journal.

\section{DISCUSSION}

This paper explains a study planned to address the issue on deprescribing in older adults with LLIs and LLE. The study proposes to gain consensus on expert opinions on a deprescribing tool for use in this population using a modified-Delphi technique. Systematically combining available evidence with expert opinion can result in 
a quality criteria or an action plan where best practice evidence is lacking. The result of the study will be used to validate the deprescribing tool that will be easy to use in the clinical setting. Previous studies utilising Delphi methodology to obtain consensus on deprescribing tools for older palliative patients ${ }^{22}$ and older frail patients ${ }^{23}$ have been developed. However, the former is specifically for cancer patients, and neither consider the complexity of deprescribing dual-purpose medications. The outcome of the present study has the potential to address the current confusion and challenge in deprescribing dual-purpose medicines in older adults with LLIs and LLE, including under palliative care. Subsequently, it will assist clinicians in reducing the medication burden in this population. This study will be using a modified-Delphi technique that is via online survey instead of a focused group discussion, which we expect to be more pragmatic and cost-effective.

This study will involve a modified-Delphi technique to gain a consensus on feedbacks from the expert panel on series of questions on the conceptual model, practicality of the tool and deprescribing of medications. The feedback will involve panel members from geographically diverse region who will rate and/or write comments on each item to elicit their opinion. The panel members will remain anonymous to each other, which will avoid individual dominance over the discussion. We consider it will take a minimum of two rounds to reach a consensus and that some participants may feel it as an exhaustive process, which is one of the demerits of this technique. However, before starting the survey, all the participants will be informed that if anytime during the Delphi process they happen to feel the burden to commit, they will always have the option to withdraw anytime without any consequence.

Deprescribing is a part of good clinical care continuum but it is often a challenging process, specifically in the older adults with LLIs and LLE. Hence, it is essential to collate information from existing knowledge and experience from experts in the geriatric field to make a meaningful decision on deprescribing in this vulnerable population. Therefore, this study design attempts to achieve a consensus on experts' opinion on a deprescribing tool for use in older adults with LLIs and LLE. We consider that this study will result in a tool that will be acceptable in clinical practice for deprescribing potentially inappropriate medications and reduce the medication burden in older adults with LLIs and LLE. In the future, the research team intends to develop this tool further for specific LLIs and to test them in a clinical setting with the appropriate population.

Contributors SS and LMN conceived the study. SS framed the conception into a protocol with assistance from AP and KJS. SS drafted and finalised the manuscript. AP, KJS and LMN supervised the work and revised the manuscript. All the authors read and approved the final version. SS and KJS drafted and revised the ethics application of the study with confirmation from AP and LMN. All the authors are accountable for all the aspects of the work.

Funding The authors have not declared a specific grant for this research from any funding agency in the public, commercial or not-for-profit sectors.
Competing interests None declared.

Patient consent for publication Not required.

Provenance and peer review Not commissioned; externally peer reviewed.

Supplemental material This content has been supplied by the author(s). It has not been vetted by BMJ Publishing Group Limited (BMJ) and may not have been peer-reviewed. Any opinions or recommendations discussed are solely those of the author(s) and are not endorsed by BMJ. BMJ disclaims all liability and responsibility arising from any reliance placed on the content. Where the content includes any translated material, BMJ does not warrant the accuracy and reliability of the translations (including but not limited to local regulations, clinical guidelines, terminology, drug names and drug dosages), and is not responsible for any error and/or omissions arising from translation and adaptation or otherwise.

Open access This is an open access article distributed in accordance with the Creative Commons Attribution Non Commercial (CC BY-NC 4.0) license, which permits others to distribute, remix, adapt, build upon this work non-commercially, and license their derivative works on different terms, provided the original work is properly cited, appropriate credit is given, any changes made indicated, and the use is non-commercial. See: http://creativecommons.org/licenses/by-nc/4.0/.

\section{ORCID iDs}

Shakti Shrestha http://orcid.org/0000-0003-0344-091X

Arjun Poudel http://orcid.org/0000-0003-3601-4650

Kathryn J Steadman http://orcid.org/0000-0001-7357-330X

Lisa M Nissen http://orcid.org/0000-0001-5826-4605

\section{REFERENCES}

1 Australian Bureau of Statistics (ABS). Australian demographic statistics. Canberra, 2017.

2 Australian Institute of Health and Welfare (AIHW). Older Australia at a glance. Canberra, 2018.

3 Australian Institute of Health and Welfare (AlHW). Deaths in Australia. Canberra, 2018.

4 Australian Institute of Health and Welfare (AlHW). Palliative care services in Australia: palliative care-related medications. Australian Insitute of Health and Welfare, 2019. https://www.aihw.gov.au/ reports/palliative-care-services/palliative-care-services-in-australia/ contents/palliative-care-related-medications

5 Poudel A, Yates P, Rowett D, et al. Use of preventive medication in patients with limited life expectancy: a systematic review. J Pain Symptom Manage 2017;53:1097-110.

6 Todd A, Husband A, Andrew I, et al. Inappropriate prescribing of preventative medication in patients with life-limiting illness: a systematic review. BMJ Support Palliat Care 2017;7:113-21.

7 Morin L, Wastesson JW, Laroche M-L, et al. How many older adults receive drugs of questionable clinical benefit near the end of life? A cohort study. Palliat Med 2019;33:0269216319854013

8 Corsonello A, Pedone C, Incalzi RA. Age-related pharmacokinetic and pharmacodynamic changes and related risk of adverse drug reactions. Curr Med Chem 2010;17:571-84.

9 Stevenson J, Abernethy AP, Miller C. Managing comorbidities in patients at the end of life. BMJ 2004;329:909-12.

10 Dees MK, Geijteman ECT, Dekkers WJM, et al. Perspectives of patients, close relatives, nurses, and physicians on end-of-life medication management. Palliat Support Care 2018;16:580-9.

11 Paque K, Vander Stichele R, Elseviers M, et al. Barriers and enablers to deprescribing in people with a life-limiting disease: a systematic review. Palliat Med 2019;33:37-48.

12 Reeve E, Gnjidic D, Long J. A systematic review of the emerging definition of 'deprescribing' with network analysis: implications for future research and clinical practice. Br J Clin Pharmacol 2015;80:1254-68.

13 Reeve E, Low L-F, Hilmer SN. Attitudes of older adults and caregivers in Australia toward deprescribing. J Am Geriatr Soc 2019;67:1204-10.

14 Ailabouni NJ, Nishtala PS, Mangin D, et al. Challenges and enablers of deprescribing: a general practitioner perspective. PLoS One 2016;11:e0151066.

15 Anderson K, Stowasser D, Freeman C. Prescriber barriers and enablers to minimising potentially inappropriate medications in adults: a systematic review and thematic synthesis. BMJ Open 2014:4:e006544.

16 Boulkedid R, Abdoul H, Loustau M. Using and reporting the Delphi method for selecting healthcare quality indicators: a systematic review. PLoS One 2011;6:e20476. 
17 SooHoo NF, Lieberman JR, Farng E. Development of quality of care indicators for patients undergoing total hip or total knee replacement. BMJ Qual Saf 2011;20:153-7.

18 American geriatrics Society 2019 updated AGS beers criteria for potentially inappropriate medication use in older adults. J Am Geriatr Soc 2019;67:674-94.

19 O'Mahony D. STOPP/START criteria for potentially inappropriate medications/potential prescribing omissions in older people: origin and progress. Expert Rev Clin Pharmacol 2020;13:15-22.

20 Farrell B, Tsang C, Raman-Wilms L. What are priorities for deprescribing for elderly patients? capturing the voice of practitioners: a modified Delphi process. Plos One 2015;10:e0122246.

21 Rodríguez-Pérez A, Alfaro-Lara ER, Albiñana-Perez S. Novel tool for deprescribing in chronic patients with multimorbidity: list of evidence-based deprescribing for chronic patients criteria. Geriatr Gerontol Int 2017;17:2200-7.

22 Lindsay J, Dooley M, Martin J, et al. The development and evaluation of an oncological palliative care deprescribing guideline: the 'OncPal deprescribing guideline'. Support Care Cancer 2015;23:71-8.

23 Curtin D, Gallagher P, O'Mahony D. Deprescribing in older people approaching end-of-life: development and validation of STOPPFrail version 2. Age Ageing 2021;50:465-71.

24 Holmes HM, Sachs GA, Shega JW, et al. Integrating palliative medicine into the care of persons with advanced dementia: identifying appropriate medication use. J Am Geriatr Soc 2008;56:1306-11.

25 Watson KE, Singleton JA, Tippett V. Defining pharmacists' roles in disasters: a Delphi study. PLoS One 2019;14:e0227132.

26 McKenna HP. The Delphi technique: a worthwhile research approach for nursing? J Adv Nurs 1994;19:1221-5.

27 Scott IA, Hilmer SN, Reeve E, et al. Reducing inappropriate polypharmacy: the process of deprescribing. JAMA Intern Med 2015;175:827-34.

28 Shrestha S, Poudel A, Steadman K, et al. Outcomes of deprescribing interventions in older patients with life-limiting illness and limited life expectancy: a systematic review. Br J Clin Pharmacol 2020;86:1931-45.

29 Akins RB, Tolson H, Cole BR. Stability of response characteristics of a Delphi panel: application of bootstrap data expansion. BMC Med Res Methodol 2005;5:37.

30 Powell C. The Delphi technique: myths and realities. J Adv Nurs 2003;41:376-82.

31 Hasson F, Keeney S, McKenna H. Research guidelines for the Delphi survey technique. J Adv Nurs 2000;32:1008-15.

32 Keeney S, Hasson F, McKenna H. Consulting the oracle: ten lessons from using the Delphi technique in nursing research. J Adv Nurs 2006;53:205-12. 